\title{
Inter-rater agreement when linking stroke interventions to the Extended International Classification of Functioning, Disability and Health core set for stroke
}

Running head: Reliability when linking interventions to the ICF

Article category: Research

Author byline: Melissa Evans, mevans@aut.ac.nz

Purpose: To uncover the factors that influence inter-rater agreement when extracting stroke interventions from patient records and linking them to the relevant categories in the Extended International Classification of Functioning, Disability and Health Core Set for Stroke.

Method: Using 10 patient files, two linkers independently extracted interventions and linked the target of the intervention to relevant functions in the ICF. The percentage agreement of extracted interventions and the ICF codes was calculated. Non-matching interventions and codes were further analysed to determine the reasons for poor agreement.

Results: 518 interventions were extracted, with $44.01 \%$ agreement between the two linkers. Of the non-agree codes and interventions, $43.79 \%$ were due to mismatched ICF codes and $56.20 \%$ were due to mismatched interventions. Differences were due to linkers a) extracting interventions from different parts of the patient note b) differences in interpreting the target of the intervention, and c) choosing a different code with similar meaning. 
Conclusion: Greater reliability when linking interventions to ICF codes can be achieved by; health services using a consistent progress note that uses ICF language, recording the intervention aim, linkers knowing the aims of each discipline's interventions and using multiple reliability checks and analysis to inform the linking method.

\section{Introduction}

The International Classification of Functioning, Disability and Health (ICF) is the World Health Organization classification system to describe human functioning and relevant environmental factors. It provides a comprehensive coverage of the functions associated with health conditions and definitions for each function and environmental factor. Relating interventions to a common reference framework identifies the health and health related domains that are addressed by the intervention, and enables different interventions to be compared across research studies and rehabilitation facilities (1). This allows services to determine the quality and equity of rehabilitation and identify whether a service is meeting the needs of specific patient groups (2).

Linking rehabilitation intervention targets to the ICF is not common in the literature, and only 5 studies have used the ICF in this way $(3,4,5,6,7)$. In the future, this method will become redundant as researchers will be able to classify interventions according to the International Classification of Health Interventions (ICHI) (2). The development of the ICHI and the digital browser will "support the production of consistently coded data" (8,p.8). However, there will be a time lag before countries and services adopt the ICHI to record and classify interventions in patient notes. In the interim, researchers can select interventions from the free text in patient notes and classify the target function of the intervention using the ICHI browser. Both these processes can cause poor reliability as researchers are required to interpret what is an 
intervention from the patient record and select the most appropriate target of the intervention.

The study reported here involved two independent linkers, who used the linking rules developed by Cieza et al. (1) to extract interventions from ten digital patient records provided by community stroke rehabilitation services. The linkers used their knowledge of the ICF and interventions for stroke to link the target function of the intervention to one or more of the 166 categories in the Extended International Core Set for Stroke (EICSS). The EICSS is validated by physicians, occupational therapists and physiotherapists, and represents the problems that are most commonly addressed by these professions $(9,10,11)$. The primary aim of this research was to investigate the inter-rater agreement between two independent linkers when extracting interventions from patient digital records, and when linking the target of the intervention to an ICF code. The secondary aims were to analyse factors that reduce inter-rater reliability; and make recommendations to improve inter-rater reliability in similar studies.

\section{Methods}

\section{Overview}

The primary researcher (ME) randomly selected 10 patient records from 113 patient records used in a larger study that explored the provision of community stroke rehabilitation to patients living in Auckland, New Zealand between 2016 and 2017. The patient's name and address were removed from the records by hospital staff before the researchers accessed a copy. Ethical approval for the study was granted by the New Zealand Health and Disability Ethics Committee and the three district health boards (DHB) that held the patient records. 
The rehabilitation team at each DHB consisted of a social worker, physiotherapist, speech language therapist, dietician, occupational therapist, nurse and rehabilitation assistants (student health professionals also contributed to the records). The ten records were from people aged 62 to 89 years (mean age 73) and 5 were male. In this group there were Māori (4), NZ European (4) and Samoan (1) and one record where the demographic data were not recorded.

\section{Data collection}

Two researchers (or linkers) involved in the study were; ME, a New Zealand educated occupational therapist (40 years since qualifying) who has worked in the stroke rehabilitation area and has had prior experience linking stroke interventions to the ICF, and CS, a UK educated physiotherapist (45 years since qualifying) who has been involved in the development and maintenance of the ICF but has limited recent experience of stroke rehabilitation.

Interventions were defined as activities in the home or community that were prescribed and supervised by a health professional or student health professional and administrative activities to access funding for equipment and home modifications. The following activities are excluded in this definition: patient assessments, meetings, referrals, and phone conversations with the patient, family, and health professionals.

\section{Data extraction and coding}

Both linkers worked independently, following the same data extraction and coding process and only meeting to discuss the non-agree codes after the results had been calculated. For each patient file, each linker extracted the date of each therapy session, the health discipline providing the intervention and the actual words from the patient 
record describing the intervention and transferred that information to an Excel spreadsheet. Linkers then selected the target of the intervention from a drop-down list in the Excel spreadsheet. The action of selecting an intervention target automatically selected an EICSS code. The list of intervention targets and pre-determined ICF codes were drawn from an earlier study by ME and colleagues that investigated interventions and targets of intervention in a similar community stroke service (6). There could be more than one target function per intervention.

To improve the consistency of coding, for difficult or ambiguous concepts, linkers were provided with a protocol showing typical interventions and their assigned codes. This protocol had been developed based on feedback given by an independent ICF expert $(\mathrm{CH})$ on samples of coding provided by ME. The use of a protocol for concepts that could be coded to two or more different codes was recommended by Cieza et al. (12), and has previously been used by researchers to improve reliability of coding $(13,14$, 15). As the 2005 linking rules were used, linkers could assign one or more targets and therefore one or more codes to an intervention. An example of extracted interventions, targets, and linked ICF code is presented in Table 1.

< Table 1. Example of extracted intervention, targets and linked ICF code>

\section{Data analysis}

Data were analysed in four stages. Stage 1 organised the data into four categories based on when linkers recorded: the same intervention and code; interventions and codes did not agree; same intervention and same ICF chapter, but the ICF code did not agree (less specific); and same intervention but did not agree at ICF chapter and code (Table 2). The data from each patient record were summed and then combined for all 10 patient 
records. An overall percentage agreement was calculated for the same interventions and codes (including chapter codes) recorded by both linkers. The criteria for acceptable percentage agreement between the two linkers was $70 \%$ (16).

In stage 2, using the extracted interventions from both linkers, the average number was calculated for: the interventions per day, the targets per ICF component (body function, activities, participation and environmental factors), and the targets per ICF chapters (sub-headings under each component). This information described the density of concepts that needed to be extracted by the linker. It is assumed that with a higher density of concepts, there will be a higher risk of mismatched interventions between the two linkers.

In stage 3 , a consensus list of interventions and codes was developed based on a consensus agreement with the other linker (CS). Following this, the extracted interventions and coding of each linker were compared to the consensus list. This analysis revealed the number of times: a) each linker had an intervention and code different to the consensus list, b) the number of times when both linkers selected the same intervention and code as the consensus list, the discrepancies in coding and whether this was due to the linker choosing an incorrect code or intervention. In stage 4, the discrepancies for each patient file and the whole data set were identified and problematic interventions and codes were analysed. (Tables 3, 4, 5 and 6).

\section{Results}

Across the 10 patient records, patients received on average 17.8 days of rehabilitation. The linkers extracted 518 interventions, with an average of 3 interventions per day from between one and six disciplines. In each treatment session, interventions on average 
were related to 1.59 components of the ICF and linked to 2 or more chapters in that component (2.27 avg.).

In comparing the results of linking interventions to ICF codes, the linkers identified the same intervention and code 228 times, resulting in a percentage agreement of 44.01. The percentage agreement increased to 51.93 when including interventions matched to the same ICF chapter, but where the specific ICF code did not agree. Of the 290 nonagree codes and interventions, $43.79 \%(\mathrm{n}=127)$ were due to mismatched codes and $56.20 \%(n=163)$ were due to mismatched interventions. Of the mis-matched codes, $32.28 \%(n=41)$ matched at the less specific chapter level and $67.71 \%(n=86)$ were mismatched. Of the mis-matched interventions $(n=163), 85$ were from linker 1 and 78 from linker 2 (Table 2).

$<$ Table 2. Numbers of agree and non-agree codes and interventions from the two linkers>

After reviewing the differences between the two linkers, ME and CS came to a consensus agreement on a selection of interventions and targets that were consistently mismatched. Following this, all the patient records were recoded based on the consensus agreement for mismatched codes and recommendations in the coding protocol. Coding that was same between the two linkers was retained. Following this, the extracted interventions and coding of each linker were compared to the consensus coding for each extracted intervention. Across the 518 codes, ME had 359 (67.91\%) and CS had $360(68.43 \%)$ codes that were the same as the consensus coding. The close alignment of the overall accuracy of the linkers indicates that no one linker was more proficient than the other. Both linkers had a similar proportion of codes that did not match the consensus coding, which indicates that the linkers had different perceptions on how to code the interventions. 


\section{Analysis of the differences in linking}

An analysis of the differences in the linkers' coding to the "consensus coding" list revealed that inconsistent extraction of interventions was caused by extracting interventions from different parts of the patient note (Table 3), linker professional bias (Table 4), and not coding the target function of the intervention (Table 5). Non-agree codes were caused by errors in coding and linkers not having clear information on the target of the intervention. This was particularly evident for interventions that had more than one target (Table 6).

<Table 3. Example of extracting interventions under different clinical note headings >

$<$ Table 4. Example of patient note showing linker bias impacting on extracted interventions>

$<$ Table 5. Differences in coding

< Table 6. Interventions coded to two or more ICF codes>

\section{Discussion}

\section{Interventions}

This study found there was poor inter-rater reliability (44.01\%) when linking 518 community stroke rehabilitation interventions to the relevant target functions in the ICF by two independent linkers. This was unexpected as both linkers had experience of 
linking health data to the ICF. In addition, efforts had been made to increase reliability by using a linking protocol and a drop-down list of intervention targets with pre-linked ICF codes. It appears that poor inter-rater reliability was largely due to linkers extracting different interventions. This was due to the following reasons:

(1) On average, there were 3.5 interventions per treatment day and of these, two were linked to a different chapter and consequently a different target function. This figure is higher than the concept density of 2.8 that was reported by Osbourne and Kauvar (15). The high number of interventions per treatment day increased the density of information for coding and may have caused linkers to miss interventions. In addition, extracting interventions was made more difficult by the presence of unfamiliar acronyms and duplicated interventions in different parts of the record.

(2) The method used to record patient notes was not consistent across professional groups and consequently the linkers had difficulty determining what part of the clinical note represented the intervention. Physiotherapists and occupational therapists used the Subjective, Objective (includes interventions), Assessment/Analysis and Plan (SOAP) format (17). Other formats were the "Subjective, Objective, Treatment, Assessment and Plan" (SOTAP) (18) and “Presentation, Client's perspective, Assessment, Intervention, Outcome and Plan". Confusion for linkers arose when the interventions were placed under headings other than Objective, Treatment and Intervention headings. This confusion is evident in Table 3 where one linker extracted information from the Objective part of the patient note and the other linker extracted interventions under Objective and Assessment. This may have occurred because the 
instructions in the study protocol stated that assessments are not considered an intervention. Nurses and social workers used a narrative approach with no clear headings. Overall, the linkers were more likely to extract the same intervention when it was written under the Objective, Treatment or Intervention headings.

(3) Professional bias was clearly seen when the linkers identified and extracted more interventions from their own discipline than from other disciplines. Although this contributed to poor inter-rater reliability, having multiple perspectives is beneficial. Professional bias can be identified by examining all non-agree codes due to linkers coding different interventions and using a consensus process to determine which interventions should be linked.

(4) When the aim of the intervention was not provided, linkers had to interpret the target based on their knowledge of the health discipline's scope of practice and the range of stroke interventions. It was not possible to clarify the target of interventions by reading the patient's initial assessments as this was not provided to linkers. The finding that poor inter-rater reliability is related to poor clarity of the text is also supported by Soberg, Sandvik (19).

(5) Non-agree interventions and coding occurred when linker 1 coded the intervention to an environmental factor code, and linker 2 coded the same intervention to a code that reflected the functional target of the intervention (Table 5). Linker 1 may have intended to capture environmental factor interventions and, in doing so, did not follow the 2019 linking rules, which state the linker should "identify the purpose of the information to be linked by answering the question: What is this information about? Or what is this item about? The answer to these questions will help identify the main concept(s)", (12,p.577). This type of issue would not have occurred if linkers had discussed 
how to interpret the aim of the intervention prior to coding or if linkers had regular meetings to compare coding of the same patient file.

(6) The Excel spread sheet used to record the intervention, and select the target of the intervention from a drop down list, may have increased the complexity of coding. This is because the wording in the drop down list was written in natural language rather than ICF terminology. In future, it is recommended linkers use their own words to describe the target of the intervention and then select a code using the ICF browser in a separate column. This process would assist researchers to monitor consistency in coding and use of the ICF browser would ensure that both linkers were using a consistent reference point.

\section{Codes}

Despite the low percentage agreement found in this study, if the non-agree interventions (163) are removed from the total number of interventions, percentage agreement to specific codes increases to $64.50 \%$.

Coding reliability was reduced when the target of the intervention could be linked to two different codes (Table 6). For example, in this research, education on fatigue management was coded by one linker to b130 energy and drive functions as the intervention target was to change the person's energy level. The other linker coded this intervention to $d 570$ looking after one's health, as it was thought that the outcome of education would assist the person to manage fatigue. The first linker has the view that the intervention aim is to address the body's capacity for energy, the second linker has the view the intervention aim is for the person to do an action that will impact on their energy use. In this example and in others in table 6 it is evident that linkers have a different view on whether an intervention target is a body function or activity and participation life area. Mismatching of codes could also be due to linkers having a 
different view on whether the target of the intervention relates to an immediate target, such as body function, or to an ultimate target in a life area. These types of coding disagreements could be reduced if therapists providing the intervention recorded the primary aim of the intervention in the patient notes.

\section{Recommendations}

To encourage consistency and to identify differences in coding between two linkers, it is recommended that linkers use an Excel spread sheet to extract the actual words for the intervention from the text, the primary target function of the intervention, and the linked ICF code using the ICF browser. To improve inter-rater reliability for extracting and linking interventions to relevant ICF codes, it is recommended that researchers do inter-rater reliability checks at the beginning of the data collection until reliability reaches an acceptable level for interventions and codes (20). After each inter-rater reliability check, differences in coding should be analysed. Researchers should check for the following causes of low reliability: interventions not being extracted from the same part of the patient note, the influence of professional bias, linkers having different views on how to interpret the target of the intervention, linkers selecting similar but different codes for the same intervention, and whether research protocols are clear to linkers. When linkers have identified different targets for same intervention, it is recommended linkers meet with the clinician and clarify the intervention target. This process may provide further insights on what wording or types of interventions cause confusion for linkers. When linkers have chosen a different but similar code for the same intervention, researchers should decide on a consistent code to be used throughout the linking process. It is natural that professional bias will be present, and researchers may consider this an asset, as different health disciplines and work experience means 
the data are seen through a variety of perspectives and there is a greater chance that all relevant interventions will be extracted.

The ICHI browser tool will now become the preferred method to classify interventions. Although this is the case, the quality of the classification process still depends on the researcher selecting the intervention from the patient's hospital records and having a sound understanding of the target of the intervention. The reliability of classifying interventions would be improved if all health disciplines used the ICF language in patient records. Where the ICF language is not used, consistency in reporting would be improved by asking services participating in the research to use a common method to record interventions that avoids the use of abbreviations and acronyms and clearly signals the target function(s) of the intervention. An example of a possible template for recording interventions is presented in table 7. Prior to classifying interventions from a health team, it may benefit the researcher to meet with each discipline and identify common interventions and the target functions of interventions.

< Table 7. Intervention template for health professional session notes>

\section{Limitations of this study}

As extracting and coding targets depends on the linker interpreting what is written, mistakes may have been due to the linker's inattention or lack of knowledge of the purpose of interventions a discipline provides. Linking was constrained to the 166 categories in the EICSS, rather than all the categories of the ICF. This may have forced linkers to use EICSS codes where another ICF code would have been more appropriate. This may have caused inconsistent coding, as linkers selected the code they considered to be the best fit with the intervention target. In future studies, it is recommended that linkers use ICF codes that are not included in the EICSS when the codes within it do not 
fully describe the intervention. No intra-rater reliability statistics were developed for this study, so it was not possible to determine whether each linker was coding the data in a consistent way. It is important to recognise that the data collected by the linkers was constrained by the composition of the rehabilitation team and the current ICF framework. In this study, the lack of psychology staff in the rehabilitation team may have impacted on the provision of interventions for emotion, stress and interpersonal and family relationships. In addition, the lack of codes for Personal Factors means that interventions directed at improving the lived experience of health were not comprehensively documented (21). This situation may soon be resolved as two recent publications have developed the definition of Personal Factors and provided codes that align with the ICF coding structure and philosophy $(21,22)$.

\section{Conclusion}

This study found there was poor inter-rater agreement (percentage agreement 44.01) when extracting and coding interventions from 10 patient files to the Extended ICF core set for stroke. Linking interventions to target functions is a complex process requiring multiple steps, which include identifying an intervention in the health record, interpreting the aim of the intervention when this is not recorded, and selecting the EICSS code(s) that best represents the intervention aim. Each step may contribute to the level of reliability between linkers. For greater reliability when using data in health records for service evaluation and planning, it is recommended that health professionals use a consistent method to record interventions and record the functional target of the intervention using ICF terminology. To eliminate factors that are causing poor interrater reliability, it is recommended that researchers conduct early robust reliability checks of extracted interventions and linked codes. 


\section{Disclosure statement}

The authors report no conflicts of interest

\section{References}

1. Cieza A, Geyh S, Chatterji S, Kostanjsek N, Üstün B, Stucki G. ICF linking rules: An update based on lessons learned. J Rehabil Med. 2005;37(4):212-8. doi: 10.1080/16501970510040263.

2. Fortune $\mathrm{N}$, Madden R, Almborg AH. Use of a new international classification of health interventions for capturing information on health interventions relevant to people with disabilities. Int J Environ Res Public Health. 2018;15(1):1-10. doi: 10.3390/ijerph15010145.

3. Boldt C, Brach M, Grill E, Berthou A, Meister K, Scheuringer M, et al. The ICF categories identified in nursing interventions administered to neurological patients with post-acute rehabilitation needs. Disabil Rehabil. 2005;27(7/8):431-6.

4. Klang Ibragimova N, Pless M, Adolfsson M, Granlund M, Björck-Åkesson E. Using content analysis to link texts on assessment and intervention to the International Classification of Functioning, Disability and Health - version for Children and Youth (ICF-CY). J Rehabil Med. 2011;43(8):728-33. doi: 10.2340/16501977-0831.

5. Tantilipikorn $P$, Watter $P$, Prasertsukdee $S$. Identifying assessment measures and interventions reported for Thai children with cerebral palsy using the ICF-CY framework. Disabil Rehabil. 2012;34(14):1178-85. doi: 10.3109/09638288.2011.637603.

6. Evans M, Hocking C, Kersten P. Mapping the rehabilitation interventions of a community stroke team to the extended International Classification of Functioning, Disability and Health Core Set for Stroke. Disabil Rehabil. 2016;November:1-7. doi: 10.1080/09638288.2016.1239763. PubMed PMID: 27866422.

7. Fitinghoff $\mathrm{H}$, Lindqvist B, Nygård L, Ekholm J, Schult ML. The ICF and postsurgery occupational therapy after traumatic hand injury. Int J Rehabil Res. 2011;34(1):79-88. doi: 10.1097/MRR.0b013e328341946c.

8. World Health Organization. ICHI beta-3 reference guide International Classification of Health Interventions 2020 [26.05.2021]. Available from: https://mitel.dimi.uniud.it/ichi/docs/. 9. Glässel A, Kirchberger I, Kollerits B, Amann E, Cieza A. Content validity of the Extended ICF Core Set for Stroke: An international delphi survey of physical therapists. Phys Ther. 2011;91(8):1211-22. doi: 10.2522/ptj.20100262.

10. Glässel A, Kirchberger I, Linseisen E, Stamm T, Cieza A, Stucki G. Content validation of the International Classification of Functioning, Disability and Health (ICF) core set for stroke: The perspective of occupational therapists. Can J Occup Ther. 2010;77(5):289-302. doi: 10.2182/cjot.2010.77.5.5.

11. Lemberg I, Kirchberger I, Stucki G, Cieza A. The ICF core set for stroke from the perspective of physicians: A worldwide validation study using the delphi technique. Eur J Phys Rehabil Med. 2010;46(3):377-88.

12. Cieza A, Fayed N, Bickenbach J, Prodinger B. Refinements of the ICF Linking Rules to strengthen their potential for establishing comparability of health information. Disabil Rehabil. 2019;41(5):574-83. doi: 10.3109/09638288.2016.1145258.

13. Van Leeuwen LM, Merkus P, Pronk M, Van Der Torn M, Maré M, Goverts ST, et al. Overlap and Nonoverlap between the ICF Core Sets for Hearing Loss and Otology and Audiology Intake Documentation. Ear Hear. 2017;38(1):103-16. doi: 10.1097/AUD.0000000000000358.

14. Granberg S, Möller K, Skagerstrand Å, Möller C, Danermark B. The ICF core sets for hearing loss: Researcher perspective, part II: Linking outcome measures to the International 
Classification of Functioning, Disability and Health (ICF). Int J Audiol. 2014;53(2):77-87. doi: 10.3109/14992027.2013.858279.

15. Osborne CL, Kauvar DS. A content analysis of peripheral arterial disease patientreported outcome measures using the International Classification of Functioning, Disability and Health. Disabil Rehabil. 2019;41(4):456-64. doi: 10.1080/09638288.2017.1390699.

16. Stemler S, Tsai J. Three best practices in interrater reliability three common approaches. In: Osborne J, editor. Best practices in quantitative methods. Thousand Oaks: SAGE Publications Inc; 2008. p. 1-45.

17. Sullivan D. Guide to clinical documentation. Philadelphia, (PA): F. A. Davis; 2018. 18. Treatment notes and progress notes using a modified SOAP format [internet]. I place unknown|: Musculoskeletal Key; 2016 [2019 December 7]. Available from:

https://musculoskeletalkey.com/treatment-notes-and-progress-notes-using-a-modified-soapformat/.

19. Soberg HL, Sandvik L, Ostensjo S. Reliability and applicability of the ICF in coding problems, resources and goals of persons with multiple injuries. Disabil Rehabil. 2008;30(2):98106. doi: 10.1080/09638280701216862.

20. Lustenberger NA, Prodinger B, Dorjbal D, Rubinelli S, Schmitt K, Scheel-Sailer A. Compiling standardized information from clinical practice: using content analysis and ICF linking rules in a goal-oriented youth rehabilitation program. Disabil Rehabil. 2019;41(5):61321. doi: 10.1080/09638288.2017.1380718.

21. Geyh S, Schwegler U, Peter C, Müller R. Representing and organizing information to describe the lived experience of health from a personal factors perspective in the light of the International Classification of Functioning, Disability and Health (ICF): a discussion paper. Disabil Rehabil. 2019;41(14):1727-38. doi: 10.1080/09638288.2018.1445302.

22. Grotkamp S, Cibis W, Brüggemann S, Coenen M, Gmünder HP, Keller K, et al. Personal factors classification revisited: A proposal in the light of the biopsychosocial model of the World Health Organization (WHO). Australian Journal of Rehabilitation Counselling. 2020;26(2):73-91. doi: 10.1017/jrc.2020.14. 
Table 1. Example of extracted intervention, target and linked ICF code

\begin{tabular}{|c|c|c|c|c|c|}
\hline Day & Date & Therapist & $\begin{array}{l}\text { Intervention } \\
\text { extracted from } \\
\text { record }\end{array}$ & Intervention target & $\begin{array}{l}\text { ICF } \\
\text { code }\end{array}$ \\
\hline 1 & $8 / 07 / 2016$ & TA & $\begin{array}{l}\text { Home exercise } \\
\text { programme completed }\end{array}$ & $\begin{array}{ll}- & \text { strength } \\
\text { - } & \text { endurance } \\
\text { - } & \text { control of } \\
& \text { voluntary } \\
& \text { movement }\end{array}$ & $\begin{array}{l}\text { b730 } \\
\text { b740 } \\
\text { b760 }\end{array}$ \\
\hline 1 & $8 / 07 / 2016$ & OT & hot drink preparation & - meal making & d630 \\
\hline 2 & $11 / 07 / 2016$ & OT & $\begin{array}{l}\text { practiced functional } \\
\text { activities that involved } \\
\text { scanning }\end{array}$ & $\begin{array}{l}\text { - undertaking a task } \\
\text { - } \quad \text { vision }\end{array}$ & $\begin{array}{l}d 210 \\
b 210\end{array}$ \\
\hline
\end{tabular}

Key: TA $=$ therapy assistant, OT $=$ occupational therapist 
Table 2. Numbers of agree and non-agree codes and interventions from the two linkers

\begin{tabular}{|c|c|c|c|c|c|c|c|c|}
\hline Patient & Inter. & $\begin{array}{r}\text { Same } \\
\text { inter. } \\
\& \\
\text { same } \\
\text { code }\end{array}$ & $\begin{array}{r}\text { Non- } \\
\text { agree } \\
\text { codes } \\
\& \\
\text { inter. }\end{array}$ & $\begin{array}{r}\text { Same } \\
\text { inter. \& } \\
\text { same } \\
\text { chapter, } \\
\text { non-agree } \\
\text { ICF code }\end{array}$ & $\begin{array}{r}\text { Same } \\
\text { inter. \& } \\
\text { non- } \\
\text { agree at } \\
\text { chapter } \\
\text { and ICF } \\
\text { code }\end{array}$ & $\begin{array}{l}\text { Non- } \\
\text { agree } \\
\text { Inter. }\end{array}$ & $\begin{array}{r}\text { New } \\
\text { Inter. } \\
\text { CS }\end{array}$ & $\begin{array}{r}\text { New } \\
\text { Inter. } \\
\text { ME }\end{array}$ \\
\hline 1 & 58 & 25 & 33 & 4 & 14 & 15 & 11 & 4 \\
\hline 2 & 69 & 31 & 38 & 1 & 6 & 31 & 22 & 9 \\
\hline 3 & 84 & 42 & 42 & 8 & 8 & 26 & 15 & 11 \\
\hline 4 & 60 & 38 & 22 & 4 & 8 & 10 & 5 & 5 \\
\hline 5 & 44 & 11 & 33 & 9 & 11 & 13 & 4 & 9 \\
\hline 6 & 13 & 5 & 8 & 1 & 2 & 5 & 5 & 0 \\
\hline 7 & 34 & 21 & 13 & 0 & 3 & 10 & 5 & 5 \\
\hline 8 & 34 & 12 & 22 & 2 & 7 & 13 & 2 & 11 \\
\hline 9 & 75 & 21 & 54 & 11 & 13 & 30 & 3 & 27 \\
\hline 10 & 47 & 22 & 25 & 1 & 14 & 10 & 6 & 4 \\
\hline TOTAL & 518 & 228 & 290 & 41 & 86 & 163 & 78 & 85 \\
\hline
\end{tabular}

Key: Inter.: interventions 
Table 3. Example of extracted interventions from different clinical note headings

\begin{tabular}{|c|c|c|}
\hline Text from clinical record & $\begin{array}{l}\text { Intervention } \\
\text { extracted: } \\
\text { Linker } 1\end{array}$ & $\begin{array}{l}\text { Intervention } \\
\text { extracted: } \\
\text { Linker 2: }\end{array}$ \\
\hline $\begin{array}{l}\text { S: patient was in back bedroom sitting in sun, said } \\
\text { she'd been out already as daughter had to go to } \\
\text { WINZ. } \\
\text { O -Alert \& agreed to participate, } \\
\text { T - Left arm exercises } \\
\text { A - Whilst sitting in chair patient was able to reach } \\
\text { to pick up water bottle from various positions on } \\
\text { floor to her left side but didn't fully extend her elbow } \\
\text { When she went to pick bottle up \& place it on table } \\
\text { she was unable to lift it far as her upper arm was too } \\
\text { sore } \\
\text { Putting a pill packet on floor was more successful as } \\
\text { patient had to reach further thereby extending her } \\
\text { elbow more until it was almost straight } \\
\text { Shoulder shrugs \& shoulder retractions were same as } \\
\text { last time and needed prompting to raise left shoulder } \\
\text { more }\end{array}$ & $\begin{array}{l}\text { Interventions } \\
\text { under the A } \\
\text { letter: } \\
\text { - picking up } \\
\text { water bottle } \\
\text { and pill } \\
\text { packet } \\
\text { (d440 fine } \\
\text { hand use) } \\
\text { - shoulder } \\
\text { shrugs and } \\
\text { shoulder } \\
\text { retractions } \\
\text { (b710 range } \\
\text { of motion) }\end{array}$ & $\begin{array}{l}\text { Intervention } \\
\text { under the T } \\
\text { letter: } \\
\text { - left arm } \\
\text { exercises } \\
\text { (d445 arm } \\
\text { and hand } \\
\text { use). }\end{array}$ \\
\hline
\end{tabular}

Key: S: subjective, O: objective, T: treatment, A: assessment. Italics: extracted interventions 
Table 4. Example of patient note showing linker bias impacting on extracted interventions

\section{Text from clinical record Interventions extracted}

S. Pt seen at home, daughter present. Plan discussed and consent gained.

O. Notable left sided inattention

T. Standing symmetry and scapular stability work at the bench, elbow extension work in weight-bearing, functional upper limb tasks; stroking the cat, turning pages of magazine and bimanual, tying shoelaces.

A. Low tone upper arm, some pain reported, requires ongoing scapular stability work and trunk rotation work.

P. Review tomorrow for Saturday high intensive visit

\author{
Both linkers extracted the \\ interventions "standing symmetry, \\ scapular stability and elbow \\ extension work in weight bearing". \\ b730 muscle power functions \\ In addition, linker 2 (occupational \\ therapist) coded "Functional upper \\ limb tasks stroking the cat, turning \\ pages of magazine and bimanual \\ tying shoelaces". \\ d445 hand and arm use \\ d540 dressing
}

Note: $\mathrm{S}=$ subjective, $\mathrm{O}=$ objective, $\mathrm{T}=$ treatment, $\mathrm{A}=$ assessment, $\mathrm{P}=$ plan

Italics: extracted interventions and linked ICF codes 
Table 5. Differences in coding

\section{Extracted intervention Linker 1 identified the $\quad$ Linker 2 identified the from clinical record environment functional aim of the intervention}

1. Set up permanent shower chair

$$
\begin{aligned}
& \text { Interpreted aim: equipment } \\
& \text { so the patient could shower } \\
& \text { Coded: e } 115 \text { products for } \\
& \text { personal use in daily living } \\
& \text { Interpreted aim: Support to } \\
& \text { the family to assist the } \\
& \text { patient with walking } \\
& \text { Coded: e } 310 \text { immediate } \\
& \text { family }
\end{aligned}
$$

2. Provided education to family on correct use of walking frame and demonstrated use

3. Brief and short Warfarin education
Interpreted aim: Education to the person on how to take medication

Coded: e110 products and substances for personal consumption
Interpreted aim: Provide chair to help with sitting

Coded: d410 changing basic body position

Interpreted aim: The patient walking with frame

Coded: d465 walking with equipment

Interpreted aim: Patient to look after health

Coded: d570 looking after one's health

Italics: indicate the different aims of the intervention given by the linkers for the same extracted intervention 
Table 6. Interventions coded to two or more ICF codes

\begin{tabular}{ll}
\hline Interventions & $\begin{array}{l}\text { Possible codes that were assigned to this } \\
\text { intervention }\end{array}$ \\
\hline Fatigue management & b130 energy and drive functions \\
& d240 handling stress \\
& d570 looking after one's health \\
Tandem stance held heel to toe for & b755 balance reactions \\
60 sec L leg leading, 30 sec on R & d410 standing \\
Walking on A and B levels with & b760 control of voluntary movement \\
eyes open and closed & b755 involuntary movement reaction functions \\
& d450 walking \\
Gardening & d6505 taking care of plants indoors and outdoors \\
& d920 recreation and leisure \\
\hline
\end{tabular}


Table 7. Intervention template for health professional session notes

\section{Intervention type Meaning of term}

Patient problem or issue:

Patient goal:

Subjective:

The subjective experience of the patient

Objective:

The clinician's objective observations and measurements

Assessment:

The clinician's interpretation of the objective section

Intervention:

The aim/target of the intervention for the patient.

Identify if the primary aim is to improve a body function, the person's ability to do a task or participate with others or to change the environment

Refer to the ICHI to identify the target of the intervention

Outcome of the

Was the intervention useful? Has the person's performance intervention: changed since the last session? How did the client/family member respond?

Plan for the next session: 